\title{
Spin-1 Model of Noninteracting Nanoparticles
}

\author{
O. YALÇIN ${ }^{a, *}$, R. $\operatorname{ERDEM}^{b}$ AND S. ÖVÜNÇ \\ ${ }^{a}$ Department of Physics, Bozok University \\ 66500, Yozgat, Turkey \\ ${ }^{b}$ Department of Physics, Gaziosmanpasa University \\ 60250, Tokat, Turkey \\ ${ }^{c}$ Decosel, Keresteciler Sitesi 12, Block No. 1 \\ Ikitelli-Istanbul, Turkey
}

(Received December 24, 2007; revised version June 6, 2008)

In this work, we presented a spin-1 model to investigate the magnetic properties of noninteracting monodomain nanoparticles based on the pair approximation. Nearest-neighbor pair interactions are incorporated between the Ising spins in three parts that are core, core-surface, and surface within the nanoparticle. Using the spin-1 Ising model of magnetization in the pair approximation, we calculated the free energy and minimized with respect to pair variables to obtain the field-cooled magnetization. Hysteresis loops of the system were plotted for various values of exchange coupling constants, and axial anisotropy of strength which couples the core, core-surface, and surface regions. The coercive field and its linear fit to the data were plotted as a function of radius of ferromagnetic nanoparticles.

PACS numbers: 75.50.Tt, 75.60.-d, 05.50.+q, 05.70.Ln

\section{Introduction}

The ferromagnetic orders in magnetic systems were dominated as monodomain (or single-domain) nanoparticles (NPs) consisting of ferromagnetic (FM) surface and antiferromagnetic (AFM) core regions which couple with each other in general $[1,2]$. In the noninteracting NPs, the FM surface and AFM core are only ordered at the lower temperatures. The magnetic properties of monodomain NPs have been attracting great interest in physics and biomedicine in recent years $[3,4]$. Firstly, the Stoner-Wohlfarth model was used in detail to describe the lowdimensional systems [5]. Magnetic evolutions for field-cooled and zero-field-cooled behavior with temperatures of a single nanoparticle were performed in various

*corresponding author; e-mail: orhanyalcin@gmail.com 
magnetic fields by using different type approximations $[1,2,6-8]$. The experimental techniques were also used for the single NP [9]. Thermodynamic properties were observed for two-dimensional magnetic NPs [10]. The first atomic-scale models of the ferrimagnetic and heterogeneous systems in which the exchange energy plays a central role in determining the magnetization of the NPs, were studied [11, 12]. A simple model has been developed to investigate magnetic nanoparticle-based systems [13]. The first magnetization measurement of individual single-domain NPs and nanowires (NWs) at very low temperature were presented [14]. Recently, magnetic structure of a NP has been investigated based on the mean-field approximation and Heisenberg Hamiltonian for a composite NP having inner FM core and outer AFM shell has been used [15]. In the above works, each of the spin sites which stand for the atomic moments in the NPs were described by the Ising spin variables that take on two values, up and down. In spite of being extensively used to calculate the magnetic properties of infinite systems, the spin-1 model in the pair approximation [16-18] has not yet been used to calculate the magnetic properties of noninteracting NPs. The studies of core-surface type NPs are extremely important for technology because of transmission of data at high density to optical computer, nanorobot to assemble, compose rigid disk. The nanoparticles have relevance to thin film devices in the new breed of magnetoelectronic, etc., spin-valve, spin-transistor and spin-dependence tunneling devices [6]. The technological applications of NPs are the focus of much research, but magnetic NPs are also used as research tools in areas of medicine, biology and materials physics.

In the scope of this work, we propose a spin-1 model to describe the magnetic properties noninteracting nanoparticles. The model is based on the pair approximation of the classical Ising-type spins $[19,20]$. Incorporating the pair correlations between the spins inside the NP, we calculated the free energy and minimized with respect to pair variables to obtain the field-cooled magnetization. Hysteresis loops, field cooling magnetization $(M)$ curves as a function of the reduced temperature and coercive field as a function of radius of NPs (or shell number) were demonstrated. The disorder of the spins at the surface induces a strongly ferromagnetic ordering of the spins in the AFM core.

\section{Theoretical model}

We consider a spherical monodomain (noninteracting) nanoparticle with the Ising spins on a hexagonal close packed (hcp) lattice for any 2D arrays which can also be extended to 3D arrays as in Fig. 1. The shells and their number originate from nearest-neighbor pair interactions in hcp lattice. In this structure, number of shells can be associated with radius $(R)$ of the NP. The radius $R$ includes number of the shells and the size of a NP increases as the number of shells increases. Therefore, we considered the Ising spins in three parts that are core (C), core-surface (CS) and surface (S) within the NP. Each of these parts 
contain core spin number $\left(N_{\mathrm{C}}\right)$, core-surface spin number $\left(N_{\mathrm{CS}}\right)$ and surface spin number $\left(N_{\mathrm{S}}\right)$, respectively. The total number of spins in a single NP involves core spin number $\left(N_{\mathrm{C}}\right)$ and surface spin number $\left(N_{\mathrm{S}}\right)$. The surface and core spins interact ferromagnetically and antiferromagnetically, respectively. This structure is made up of three shells, namely $\mathrm{C}, \mathrm{CS}$ and $\mathrm{S}$ regions.

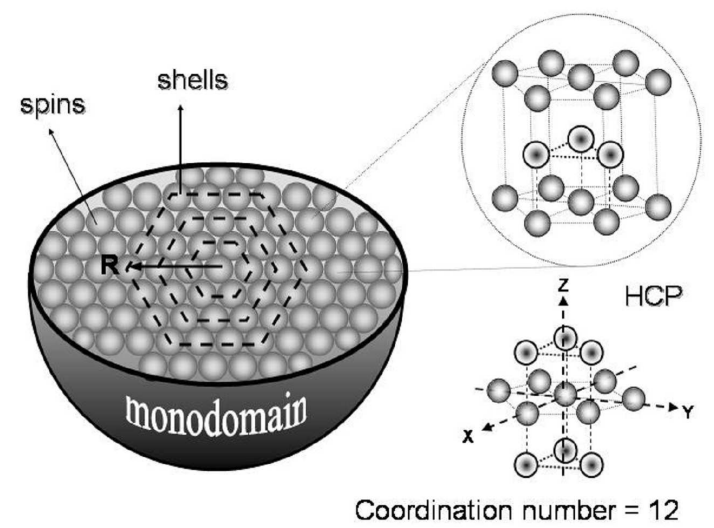

Fig. 1. A spherical monodomain magnetic NP spaced coherently in a form of 3D array. The shape of a single NP consists of the hexagonal lattice. The dashed lines displayed shells of spins in two-dimensional finite array. The radius of NPs $(R)$ includes shell numbers. Inset in this figure exhibits coordination number of hcp structure.

The magnetic particles become single domain below a critical size in contrast with the usual multidomain structure of the bulk magnetic materials. Therefore, in the scope of this work, we will study magnetic properties of monodomain NPs manifestations and only consider spin model Hamiltonians. The spin-1 model Hamiltonian for homogeneous NPs is given by

$$
\mathcal{H}=-\sum_{\langle i, j\rangle} J_{i j} S_{i} S_{j}-H \sum_{i}^{N} S_{i},
$$

where the spins $S_{i}$ located at sites $i$ on a discrete lattice can take the values \pm 1 , $0, N$ is the total number of lattice points in NPs, $H$ is the external magnetic field and $J_{i j}$ is the exchange coupling between nearest-neighbor spins inside the NP. If $J_{i j}>0$, parallel orientation of the spins is favored and at the low temperatures all spins will be aligned ferromagnetically. For $J_{i j}<0$, the low temperature phase is AFM with the spins aligned antiparallel. Equation (1) describes a paramagnetic character at all temperatures when $J_{i j}=0$. The single-ion anisotropy is implicitly included in the classical theoretical models of NPs according to the experimental results [6]. Also, the anisotropy constant is usually small and anisotropy energy contribution is considered as perturbation in quantum mechanical theories of NPs [15]. Therefore, we neglected one-ion anisotropy constant in our classical Hamil- 
tonian. Having defined the relevant spins that describe the system we start by writing the spin-1 model Hamiltonian with exchange interactions for core $\left(J_{\mathrm{C}}\right)$, core-surface $\left(J_{\mathrm{CS}}\right)$ and surface $\left(J_{\mathrm{S}}\right)$ regions (or composite NPs),

$$
\mathcal{H}=-\sum_{\langle i, j\rangle} J_{\mathrm{C}} S_{i} S_{j}-\sum_{\langle i, j\rangle} J_{\mathrm{CS}} S_{i} S_{j}-\sum_{\langle i, j\rangle} J_{\mathrm{S}} S_{i} S_{j}-H \sum_{i}^{N} S_{i},
$$

where $\langle i, j\rangle$ denotes a nearest-neighbor pair of lattice sites for each regions (core, core-surface, and surface). Spin-1 model is a three-state system and the average value of each of the spin states will be indicated by $X_{1}, X_{2}$ and $X_{3}$, which are also called the state variables or point variables. $X_{1}, X_{2}$, and $X_{3}$ are the average fractions of the spins with values $+1,0$ and -1 , respectively. These variables obey the following normalization relation:

$$
\sum_{i=1}^{3} X_{i}=1
$$

In order to consider the pair correlation, we introduce the internal variables $Y_{i j}$, indicating the average number of the states in which the first member of the nearest-neighbor pair is in state $i$ and the second member in state $j$. These will be called pair or bond variables interchangeably. The relations between $X_{i}$ and $Y_{i j}$ are as follows:

$$
X_{i}=\sum_{j=1}^{3} Y_{i j} \quad(i=1,2,3) .
$$

If the number of pairs in the system is $N_{\mathrm{p}}$, the number of $(+,+)$ bonds in the system is $Y_{11} N_{\mathrm{p}},(0,0)$ bonds, $Y_{22} N_{\mathrm{p}},(-,-)$ bonds, $Y_{33} N_{\mathrm{p}},(+, 0)$ bonds, $Y_{12} N_{\mathrm{p}}$, $(0,+)$ bonds, $Y_{21} N_{\mathrm{p}},(+,-)$ bonds, $Y_{13} N_{\mathrm{p}},(-,+)$ bonds, $Y_{31} N_{\mathrm{p}},(0,-)$ and $(-, 0)$ bonds in the system are $Y_{23} N_{\mathrm{p}}$ and $Y_{32} N_{\mathrm{p}}$, respectively. The $Y_{i j}$ 's are normalized by the equations

$$
\sum_{i, j=1}^{3} Y_{i j}=1
$$

The average magnetization $\langle M\rangle$, which is the excess of one orientation over the other orientation and also called the dipole moment, is given by

$$
M=\langle M\rangle=X_{1}-X_{3}=Y_{11}+Y_{12}+Y_{13}-\left(Y_{31}+Y_{32}+Y_{33}\right) .
$$

The interaction energy $E$ can be written in terms of $Y_{i j}$ as

$$
\beta E=N_{\mathrm{C}} \frac{\gamma}{2} \sum_{i, j}^{3} \eta_{j i} Y_{i j}+N_{\mathrm{CS}} \frac{\gamma}{2} \sum_{i, j}^{3} \eta_{j i} Y_{i j}+N_{\mathrm{S}} \frac{\gamma}{2} \sum_{i, j}^{3} \eta_{j i} Y_{i j}, \quad E=\langle H\rangle,
$$

where $\beta=1 / k_{\mathrm{B}} T . N_{\mathrm{C}}, N_{\mathrm{CS}}, N_{\mathrm{S}}$ are the numbers of lattice points in core, coresurface, and surface area, respectively. $\gamma$ is the number of nearest neighbors for a given lattice, or the coordination number of the lattice and where (all over the $\eta_{i j}$ expression in NP combined each other) 


$$
\begin{aligned}
& \eta_{11}=-J_{\mathrm{C}}-J_{\mathrm{CS}}-J_{\mathrm{S}}-H, \quad \eta_{22}=0, \quad \eta_{33}=-J_{\mathrm{C}}-J_{\mathrm{CS}}-J_{\mathrm{S}}+H, \\
& \eta_{12}=-H, \quad \eta_{21}=0, \quad \eta_{13}=J_{\mathrm{C}}+J_{\mathrm{CS}}+J_{\mathrm{S}}-H, \\
& \eta_{31}=J_{\mathrm{C}}+J_{\mathrm{CS}}+J_{\mathrm{S}}+H, \quad \eta_{23}=0, \quad \eta_{32}=H .
\end{aligned}
$$

The number of ways in which independent pairs can be arranged on the lattice is

$$
W_{\mathrm{p}}=\frac{\left(\gamma N_{\mathrm{C}} / 2\right) !}{\prod_{i, j=1}^{3}\left(\gamma N_{\mathrm{C}} Y_{i j} / 2\right) !} \cdot \frac{\left(\gamma N_{\mathrm{CS}} / 2\right) !}{\prod_{i, j=1,3}^{3}\left(\gamma N_{\mathrm{CS}} Y_{i j} / 2\right) !} \cdot \frac{\left(\gamma N_{\mathrm{S}} / 2\right) !}{\prod_{i, j=1,3}^{3}\left(\gamma N_{\mathrm{S}} Y_{i j} / 2\right) !},
$$

since there are $N_{\mathrm{p}, \mathrm{C}}=\gamma N_{\mathrm{C}} / 2, N_{\mathrm{p}, \mathrm{CS}}=\gamma N_{\mathrm{CS}} / 2$ and $N_{\mathrm{p}, \mathrm{S}}=\gamma N_{\mathrm{S}} / 2$ pairs. In terms of $X_{i}$ the number of ways the spins can be arranged is

$$
W_{x}=\frac{N_{\mathrm{C}} !}{\prod_{i=1}^{3}\left(X_{i} N_{\mathrm{C}}\right) !} \cdot \frac{N_{\mathrm{CS}} !}{\prod_{i=1}^{3}\left(X_{i} N_{\mathrm{CS}}\right) !} \cdot \frac{N_{\mathrm{S}} !}{\prod_{i=1}^{3}\left(X_{i} N_{\mathrm{S}}\right) !} .
$$

For this system the entropy and the free energy are given by

$$
S=k_{\mathrm{B}} \ln W, \quad F=E-T S,
$$

where $S=S_{\mathrm{C}}+S_{\mathrm{CS}}+S_{\mathrm{S}}$. Using Eqs. (9)-(11) and making use of the Stirling approximation, the free energy $\phi$ can be found as [16-18]:

$$
\begin{aligned}
\phi= & \beta F=N_{\mathrm{C}} \frac{\gamma}{2} \sum_{i, j}^{3} \eta_{i j} Y_{i j}+N_{\mathrm{C}} \frac{\gamma}{2} \sum_{i, j}^{3} Y_{i j} \ln \left(Y_{i j}\right)+N_{\mathrm{CS}} \frac{\gamma}{2} \sum_{i, j}^{3} \eta_{i j} Y_{i j} \\
& +N_{\mathrm{CS}} \frac{\gamma}{2} \sum_{i, j}^{3} Y_{i j} \ln \left(Y_{i j}\right)+N_{\mathrm{S}} \frac{\gamma}{2} \sum_{i, j}^{3} \eta_{i j} Y_{i j}+N_{\mathrm{S}} \frac{\gamma}{2} \sum_{i, j}^{3} Y_{i j} \ln \left(Y_{i j}\right) \\
& -(\gamma-1) \sum_{i=1}^{3} X_{i} \ln \left(X_{i}\right)+\beta \lambda\left(1-\sum_{i, j=1}^{3} Y_{i j}\right),
\end{aligned}
$$

where $\lambda$ is introduced to maintain the normalization condition for each regions (core, core-surface, and surface). The next to the last term includes the correction of the overcount of the pair term. The free energy for a system at equilibrium is a minimum state. The minimization of Eq. (9) with respect to $Y_{i j}$ leads to the following nine self-consistent equations for the pair variables:

where

$$
Y_{i j}=\frac{1}{Z}\left(X_{i} X_{j}\right)^{\bar{\gamma}} \mathrm{e}^{-\eta_{i j}} \equiv \frac{e_{i j}}{Z},
$$

$$
\bar{\gamma}=\frac{\gamma-1}{\gamma}, \quad Z=\exp (2 \beta \lambda / \gamma)=\sum_{i, j=1}^{3} e_{i j} .
$$

These nine self-consistent equations can be written explicitly by using Eq. (8) in Eq. (13) as,

$$
\begin{aligned}
Y_{11} & =\frac{1}{Z}\left(X_{1} X_{1}\right)^{\bar{\gamma}} \exp \frac{\gamma}{2}\left(-N_{\mathrm{C}} J_{\mathrm{C}}-N_{\mathrm{CS}} J_{\mathrm{CS}}-N_{\mathrm{S}} J_{\mathrm{S}}-N H\right) \equiv \frac{e_{11}}{Z}, \\
Y_{22} & =\frac{1}{Z}\left(X_{2} X_{2}\right)^{\bar{\gamma}} \equiv \frac{e_{22}}{Z}
\end{aligned}
$$




$$
\begin{aligned}
Y_{33} & =\frac{1}{Z}\left(X_{3} X_{3}\right)^{\bar{\gamma}} \exp \frac{\gamma}{2}\left(-N_{\mathrm{C}} J_{\mathrm{C}}-N_{\mathrm{CS}} J_{\mathrm{CS}}-N_{\mathrm{S}} J_{\mathrm{S}}+N H\right) \equiv \frac{e_{33}}{Z} \\
Y_{12} & =\frac{1}{Z}\left(X_{1} X_{2}\right)^{\bar{\gamma}} \exp \frac{\gamma}{2}(-N H) \equiv \frac{e_{12}}{Z} \\
Y_{21} & =\frac{1}{Z}\left(X_{2} X_{1}\right)^{\bar{\gamma}} \equiv \frac{e_{21}}{Z} \\
Y_{13} & =\frac{1}{Z}\left(X_{1} X_{3}\right)^{\bar{\gamma}} \exp \frac{\gamma}{2}\left(N_{\mathrm{C}} J_{\mathrm{C}}+N_{\mathrm{CS}} J_{\mathrm{CS}}+N_{\mathrm{S}} J_{\mathrm{S}}-N H\right) \equiv \frac{e_{13}}{Z} \\
Y_{31} & =\frac{1}{Z}\left(X_{3} X_{1}\right)^{\bar{\gamma}} \exp \frac{\gamma}{2}\left(N_{\mathrm{C}} J_{\mathrm{C}}+N_{\mathrm{CS}} J_{\mathrm{CS}}+N_{\mathrm{S}} J_{\mathrm{S}}+N H\right) \equiv \frac{e_{13}}{Z} \\
Y_{23} & =\frac{1}{Z}\left(X_{2} X_{3}\right)^{\bar{\gamma}} \equiv \frac{e_{23}}{Z}, \\
Y_{32} & =\frac{1}{Z}\left(X_{3} X_{2}\right)^{\bar{\gamma}} \exp \frac{\gamma}{2}(N H) \equiv \frac{e_{32}}{Z}
\end{aligned}
$$

where $N=N_{\mathrm{C}}+N_{\mathrm{S}}$. Above nine self-consistent equations are solved using the Newton-Raphson method for hcp lattice with coordination numbers $\gamma=6$ in 2D or $\gamma=12$ in 3D. After establishing the $Y_{i j}$ values the field cooling magnetization $(M)$ values can be obtained easily using Eq. (6). The temperature and magnetic field dependences of $M$ are shown in Figs. 2, 3, and 4, respectively. The coercive field $\left(H_{\mathrm{C}}\right)$ and its linear fit to the data as a function of diameter are also plotted in Fig. 3, and $H_{\mathrm{C}}$ vs. reduced temperature is obtained by using the hysteresis loops in Fig. 5 .

\section{Results and discussion}

For the evolution of the normalized field-cooled magnetization $(M)$ as a function of the reduced temperature $\left(k_{\mathrm{B}} T / J_{0}\right)$, we consider a six-shell NP whose core is predominantly AFM, $J_{\mathrm{C}}=-J_{0} / 2$ for $R=0-5$, with a FM surface, $J_{\mathrm{S}}=2 J_{0}$ for $R=6$, which is ferromagnetically coupled with the core, $J_{\mathrm{CS}}=J_{0}$ between $R=5$ and $R=6$ shells. The energy parameter is $J_{0}=1$. The core includes the central spin and all spins up to the fifth shell for $R=6$, i.e., $N_{\mathrm{C}}=91$, the surface has $N_{\mathrm{S}}=36$ spins and CS region has $N_{\mathrm{CS}}=33$ spins. The ratio for six shells of NPs between the surface and core spins is around 0.395. Using Eq. (6) we plot $M$ vs. $k_{\mathrm{B}} T / J_{0}$ in Fig. 2 for the above values of the parameters. As seen from this figure, the changes in the magnetization with temperature also point out interesting aspects. The magnetization curves are increasing from zero to +1 value while the reduced temperature is decreasing. In additional, the total magnetization of noninteracting NPs is slowly decreasing while magnetic field is increasing according to the reduced temperature. The Curie temperature is decreasing as the magnetic field is decreasing. In other words, the transition temperature in lower magnetic field is significantly sharp compared to that for the transition temperature in high magnetic field. It is interesting that for a very small magnetic field (and very small NPs) a well-defined phase transition occurs. It can be seen from this figure that the transition corresponds to a second-order phase transition. 


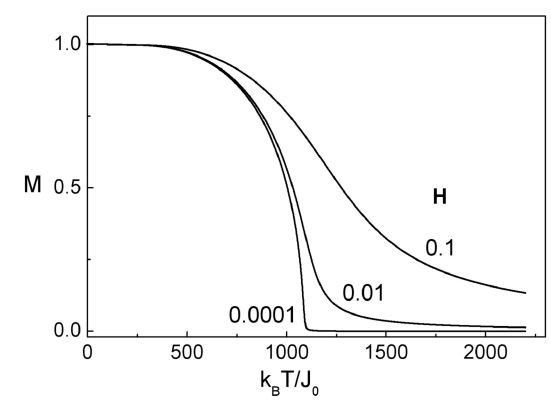

Fig. 2. The temperature evolution of normalized magnetization for field cooling in six shells. Different values for the applied magnetic field are considered. The monodomain NPs consist of $R=6$ shells for $N_{\mathrm{C}}=91, N_{\mathrm{S}}=36, N_{\mathrm{CS}}=33$ in $2 \mathrm{D} . J_{0}=1, J_{\mathrm{C}}=-J_{0} / 2$, $J_{\mathrm{S}}=2 J_{0}, J_{\mathrm{CS}}=J_{0}$.
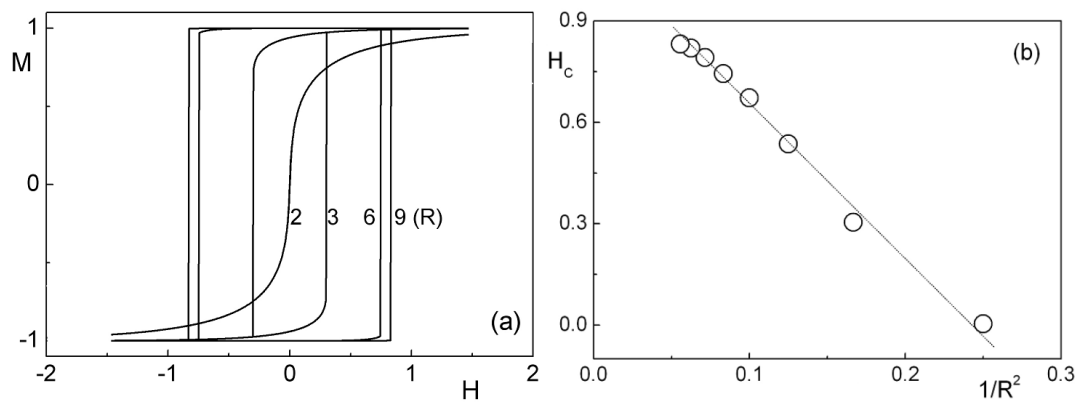

Fig. 3. (a) Hysteresis loops for various sizes of homogeneous NPs: $R=2$ with $N_{\mathrm{C}}=7$, $N_{\mathrm{S}}=12, N_{\mathrm{CS}}=9 ; R=3$ with $N_{\mathrm{C}}=19, N_{\mathrm{S}}=18, N_{\mathrm{CS}}=15 ; R=6$ with $N_{\mathrm{C}}=91$, $N_{\mathrm{S}}=36, N_{\mathrm{CS}}=33$ and $R=9$ with $N_{\mathrm{C}}=217, N_{\mathrm{S}}=54, N_{\mathrm{CS}}=51$ in $2 \mathrm{D} . T=300 J_{0} / k_{\mathrm{B}}$, $J_{0}=1, J_{\mathrm{C}}=J_{\mathrm{S}}=J_{\mathrm{CS}}=J_{0}$. (b) The coercive field plotted as a function of $R^{-2}$. The open circles correspond to $R=2,3, \ldots, 9$ and the straight line results from a linear fit to the data.

The magnetic field evolutions (or hysteresis loops) of normalized magnetization and its coercive field of the noninteracting homogeneous NPs for various diameters are given in Fig. 3. We consider a FM coupling in surface $\left(J_{\mathrm{S}}=J_{0}\right)$, core $\left(J_{\mathrm{C}}=J_{0}\right)$ and core-surface $\left(J_{\mathrm{CS}}=J_{0}\right)$ regions with $J_{0}=1$ on a hexagonal lattice. The hysteresis curves of small diameter NPs are approximately the same. This behavior is called superparamagnetic (SP) regime. However, loops strongly depend on the size (diameter) of NP and the hysteresis curves in high diameter values change sharply, as shown in Fig. 3a. Moreover, the hysteresis loops are broadening while the diameter of NP is increasing so that it approaches to bulk materials. The size dependence of the coercive field $H_{\mathrm{C}}$ is plotted as determined from the magnetization loops in Fig. 3b. In the figure, the open circles correspond to $R=2,3 \ldots 9$ and the straight line are the results from a linear fit to the data. 
The coercive field depends linearly on $R^{-2}$. While the magnetic field increases, $H$ reaches $H_{\mathrm{C}}$.

Magnetic hysteresis loops of composite NPs for various sizes with $R=$ $2,3,4,6,10$ and for some selected temperatures with $R=3$ shells, $N_{\mathrm{C}}=19$, $N_{\mathrm{CS}}=15, N_{\mathrm{S}}=18$ are shown in Fig. 4 . The exchange interactions in the surface and core regions are given by the coupling parameters $J_{\mathrm{S}}=5 J_{0}$ and $J_{\mathrm{C}}=J_{0}$ $\left(J_{0}=1\right)$, respectively, and the coupling between core and surface is due to an AFM exchange constant $J_{\mathrm{CS}}=-4 J_{0}$. From the figure, it is clear that there is no correlation between the inner FM core and outmost FM surface. Each spin in the core and surface regions can be seen as an average interaction with all the other spins. But, hysteresis curves strongly depend on the size of composite NPs, as seen in Fig. 4a. The loops in high diameter values change suddenly. This behavior originates from bulk materials. We consider a AFM coupling between core and surface when FM interactions exist on both surface and core. These hysteresis curves are plotted easily using Eq. (6). As shown in Fig. 4b, magnetization changes with magnetic field near the zero value from -1 to +1 . The evolutions of hysteresis loops of composite NPs are seen to change monotonically as the temperature increases. Figure $4 \mathrm{~b}$ also shows the hysteresis curves of NPs in SP regime at $550 k_{\mathrm{B}} T$ and ferromagnetism between $150-450 k_{\mathrm{B}} T$. In the inset, temperature dependence of the coercivity $\left(H_{\mathrm{C}}\right)$ is determined from magnetization loops in this figure. The coercivity and SP transition temperatures exhibit important aspects in the future high-density magnetic data storage.

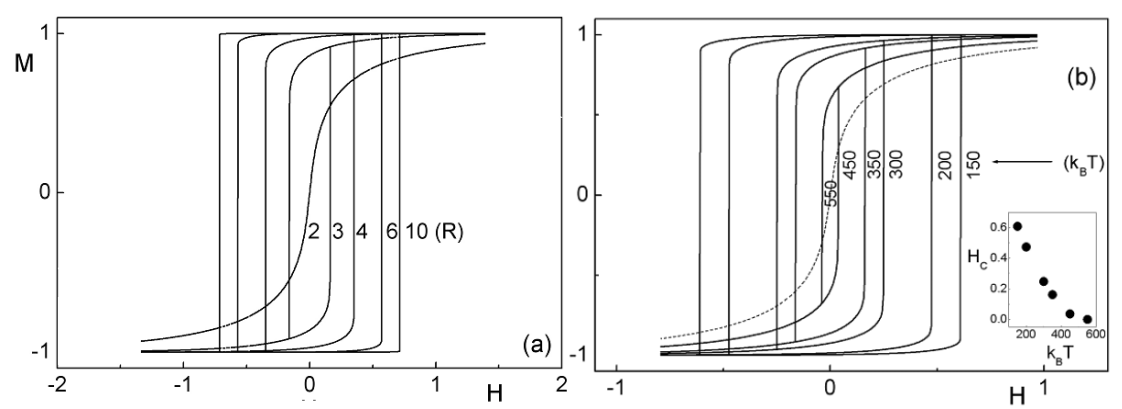

Fig. 4. (a) Hysteresis loops for various sizes of composite NPs: $R=2 ; 3 ; 4$ with $N_{\mathrm{C}}=37, N_{\mathrm{S}}=24, N_{\mathrm{CS}}=21 ; 6$ and 10 with $N_{\mathrm{C}}=271, N_{\mathrm{S}}=60, N_{\mathrm{CS}}=57$ in $2 \mathrm{D}$. $T=350 J_{0} / k_{\mathrm{B}}, J_{0}=1, J_{\mathrm{S}}=5 J_{0}, J_{\mathrm{C}}=J_{0}, J_{\mathrm{CS}}=-4 J_{0}$. (b) The same as Fig. 4a but for different temperatures of composite NP with $R=3$ shells and in the inset temperature dependence of the coercivity is determined from magnetization loops in this figure.

Finally, the evolution of the hysteresis loops for various sizes $(R=2,3,4,6$, 8, and 10) of composite NPs are given in 2D in Fig. 5. In this figure, the reduced temperature and exchange constants are $T=350 J_{0} / k_{\mathrm{B}}, J_{0}=1, J_{\mathrm{C}}=-J_{0} / 2$, $J_{\mathrm{CS}}=J_{0}$ and $J_{\mathrm{S}}=2 J_{0}$, respectively. The dotted line and full line correspond, 


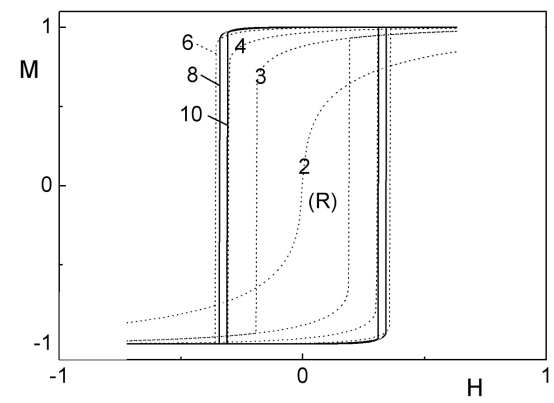

Fig. 5. Hysteresis loops for various sizes of composite NPs: $R=2,3,4,6,8$, and 10 in $2 \mathrm{D}$. Here $R=2,3,4,6$, and 10 shells have the same number of spins as in Fig. 4 . The number of spins for core, surface and core-surface regions with $R=8$ shells are $N_{\mathrm{C}}=169, N_{\mathrm{S}}=48, N_{\mathrm{CS}}=45$, respectively. $T=350 J_{0} / k_{\mathrm{B}}, J_{0}=1, J_{\mathrm{C}}=-J_{0} / 2$, $J_{\mathrm{CS}}=J_{0}, J_{\mathrm{S}}=2 J_{0}$.

respectively, to broadening and narrowing loops with increasing size of NP. The AFM nanoparticles could exhibit SP relaxation of their spin lattices as well as the permanent moments arising from uncompensated surface spins. As evidenced by this figure, the hysteresis curves are dramatically changed with increasing radius between $R=6$ and $R=8$. This change is reasonable to anticipate the same effect as a result of the exchange interaction between the uncompensated FM surface and AFM core of the particles. In other words, AFM core is dominating according to the FM surface in NPs. The quantum effects and experimental results make the NPs system very exciting for future investigations.

\section{Conclusion}

In this work, we focus on the magnetic properties of the noninteracting homogeneous ferromagnetic NPs and composite NPs having inner AFM (and FM) core, a FM (and AFM) coupling between core and surface and outer FM surface for various diameters and temperatures. As seen from Figs. 3a, 4a, and 4b, the magnetic behavior is proportional with diameter of NPs and inversely proportional with temperature, respectively. The magnetic properties of monodomain NPs have a marked dependence on the particle size and the surface effects start to dominate. In noninteracting NPs having large ratio of surface to volume, the spin disorder may modify the magnetic properties. Supposing the number of core atoms is $N_{\mathrm{C}}$, the number of surface atoms according to the number of core atoms is $(1 / 4) N_{\mathrm{C}}$ for individual NPs. The spin disorder can be caused by lower coordination of the surface atoms, broken exchange interactions that produce spin-glass like state of spatially disordered spin in the surface captions with inhomogeneous surface effects [21-23]. When the diameter of the NPs decrease, the overall behaviors can be changed. At high temperatures and small size of nanoparticles, hysteresis is observed as expected in the SP behavior. With increase in the particle size the Curie temperature approaches the bulk materials' Curie temperature which 
is a consistent result with those of the site-dependent mean-field approach for the Heisenberg spins developed in Ref. [15]. Below the Néel temperature, the outer AFM surface and inner FM core gets ordered. The size effect of small monodomain NPs is observed and reduction in the number of the surface spin causes fundamental changes in the magnetic order. The magnetic properties of NPs from this work are quite different from bulk materials. It is clear that the transition temperature of NPs is lower than that of corresponding bulks materials. Magnetic properties of NPs can be described by assuming that all the magnetic moments are rigid as a single "giant" spin. This property is the essence of the superparamagnetism.

\section{References}

[1] L.G. Rego, W. Figueiredo, Phys. Rev. B 64, 44424-1 (2001).

[2] V.S. Leite, W. Figueiredo, Brazilian J. Phys. 34, 452 (2004).

[3] A.A. Fraerman, S.A. Gusev, I.M. Nefedov, Y.N. Nozdrin, I.R. Karetnikova, L.A. Mazo, M.V. Sapozhnikov, I.A. Shereshevsky, L.V. Suhodoev, J. Phys., Condens. Matter 13, 683 (2001).

[4] Q.A. Pankhurst, J. Connolly, S.K. Jones, J. Dobson, J. Phys. D, Appl. Phys. 36, R167 (2003).

[5] E.C. Stoner, E.P. Wohlfarth, Philos. Trans. R. Soc. Lond. Ser. A 240, 599 (1948); reprinted by IEEE Trans. Magn. 27, 3475 (1991).

[6] V. Babin, P. Garstecki, R. Holyst, J. Appl. Phys. 94, 4244 (2003).

[7] M.G. del Muro, X. Batlle, A. Labarta, Phys. Rev. B 59, 13584 (1999).

[8] A. Szlaferek, Phys. Status Solidi B 241, 1312 (2004).

[9] W. Werndorfer, D. Mailly, A. Benoit, J. Appl. Phys. 87, 5094 (2000).

[10] P. Vargas, D. Altbir, M. Knobel, D. Laroze, Europhys. Lett. 58, 603 (2002).

[11] R.H. Kodama, A.E. Berkowitz, Phys. Rev. B 59, 6321 (1999).

[12] R.H. Kodama, A.E. Berkowitz, E.J. McNiff, Jr., S. Foner, Phys. Rev. Lett. 77, 394 (1996).

[13] A.F. Bakuzis, P.C. Morais, J. Magn. Magn. Mater. 272-276, e1161 (2004).

[14] W. Wernsdorfer, K. Hasselbach, D. Mailly, B. Barbara, A. Benoit, L. Thomas, G. Suran, J. Magn. Magn. Mater. 145, 33 (1995).

[15] N.A. Usov, S.A. Gudoshnikov, J. Magn. Magn. Mater. 290-291, 727 (2005).

[16] M. Keskin, P.H.E. Meijer, J. Chem. Phys. 85, 7324 (1986).

[17] M. Keskin, A. Erdinç, Tr. J. Phys. 19, 88 (1995).

[18] A. Erdinç, M. Keskin, Physica A 307, 453 (2002).

[19] R. Kikuchi, J. Chem. Phys. 60, 1071 (1974).

[20] R. Kikuchi, Crystals Statistics, Hughes Research Lab. Malibu, CA 1979, unpublished results.

[21] R.H. Kodama, J. Magn. Magn. Mater. 200, 359 (2005).

[22] V.S. Leite, W. Figueiredo, Physica A 350, 379 (2005).

[23] T. Kaneyoshi, Phys. Status Solidi B 242, 2938 (2005). 